\title{
Effect of surface structure on peptide adsorption on soft surfaces
}

\author{
David L. Cheung* \\ School of Chemistry, National University of Ireland Galway, University Road, Galway, Ireland
}

\begin{abstract}
Understanding the adsorption of proteins onto material surfaces is a major challenge in the design of biomaterials. As the behaviour of proteins nears surfaces depends on the surface chemistry and structure, along with the protein sequence, a microscopic understanding of the protein adsorption process requires insight into the interplay between these different effects. Knowledge of how the nanoscale surface structure affects protein adsorption is in particular lacking. Using molecular dynamics simulations the effect of changing surface structure, specifically the introduction of alternating hydrophobic and hydrophilic stripes, on the adsorption of model peptides is investigated. The strongest adsorption is found for surfaces with larger hydrophobic regions, as the peptides can minimise unfavourable contacts with hydrophilic regions of the surface, while the adsorption strength decreases with the stripe width, becoming comparable to that of a purely hydrophilic surface for the thinnest stripes. Changes to the conformational entropy of the peptides during adsorption are shown to play an important role in controlling the adsorption strength, with differences between the peptides appearing for narrow stripes. This information may be used to understand how the adsorption of proteins is related to the surface structure, allowing for control over biocompatibility and anti-fouling behaviour,
\end{abstract}

\section{INTRODUCTION}

Adsorption of proteins onto material surfaces is the initial event that occurs when a synthetic object comes into contact with a biological system. As this often triggers an immune response that dictates the eventual fate of the system, understanding the interaction between the material surface and proteins is vital for the design of novel materials to be used in medical devices, implants and prosthetics. Much effort has been used in the pursuit of materials that resist the adsorption of proteins ${ }^{1,2}$. There has been particular interest the development of soft surfaces, such as self-assembled monolayers ${ }^{3}$ (SAM) or polymer brushes ${ }^{4}$, as their ease of modification allows them to be tailored for specific applications. Considerable synthetic effort has been expended over a number of years and a variety of protein resistant surfaces have been developed. A few guidelines for the design of biocompatible surfaces have been proposed ${ }^{5}$ (e.g. charged, hydrophilic materials) but exceptions to these exist ${ }^{6}$, so understanding the basis for the design of biocompatible surface coatings is an on going concern.

As well as different material, changes to the surface structure many also affect the adsorption of proteins. This is common in biological systems, where biointerfaces, such as the cell membrane, are often structured on nanometre lengthscales. One synthetic approach involves the use of mixtures of positively and negatively charged molecules, leading to a zwitterionic surface ${ }^{7}$. This leads to strong water structuring and the charge pairing reduces the surface dipole moment, both factors that are expected to reduce protein adsorption ${ }^{8}$. There has also been interest in the creation of surfaces containing incompatible ligands, such as mixtures of hydrophilic and hydrophobic molecules ${ }^{9,10}$, or hydrocarbon- fluorocarbon mixtures ${ }^{11}$. The differing chemistry of the molecules may give these surfaces characteristics that may be significantly different to uniform ones ${ }^{12,13}$. However, it can be difficult to control demixing between different types of ligand molecules, so large-scale preparation of well-defined surfaces is difficult. This makes it experimentally difficult to relate protein adsorption to the nanoscale topology. Recently the use bidentate ligands with both hydrophobic and hydrophilic end groups has allowed for the construction of surfaces with incompatible ligands with controlled nanoscale topology ${ }^{14-16}$, with feature sizes on the molecular lengthscales. As this is comparable in size to proteins it may be expected that the interaction between these surfaces and biomolecules may be significantly different to uniform surfaces. Indeed properties of such structured surfaces, such as the surface free energy, depend on their structure in a nontrivial manner ${ }^{17}$. Changing the sizes of the surface features therefore provides an alternative approach to control their interactions with biomolecules.

While a range of experimental methods have been applied to the study of protein adsorption onto surfaces, the limited time and spatial resolution of these means that knowledge of the initial stages of protein adsorption is still lacking. Molecular simulation operates directly on the molecular level and so can give microscopic information regarding the behaviour of proteins at surfaces, such as determining adsorption strengths of proteins ${ }^{18,19}$ and identifying key residues involved in the adsorption process $^{20}$. It has been used to investigate adsorption of proteins onto a range of different surfaces ${ }^{21,22}$, including metals, inorganic materials, and polymer surfaces. A number of studies of protein adsorption onto selfassembled monolayers have been performed ${ }^{18,19,23-28}$, demonstrating that with appropriate force fields and simulation techniques adsorption free energies in good agreement with experimental can be obtained. In particular the importance of adequately sampling the protein conformations near the surface has been demonstrated ${ }^{18,19}$. The changes in protein conformation at interfaces, necessary for understanding the subsequent formation of inter- 
facial layers has also been investigated for small proteins. While previous studies have largely focused on uniform monolayers a few simulations of protein adsorption onto mixed SAMs have been performed, including the investigation of cytochrome-C on mixed monolayer protected nanoparticles ${ }^{29}$ and the hydrophobin EAS adsorbed on nanopatterned surfaces ${ }^{30}$. These studies have given insight into the effect of changing surface patterning on protein adsorption, in particular identifying the role played by the amphipathic character of lysine residues. However, as these focused on specific experimental systems they were unable to give insight into the generic effect of surface structure and protein adsorption. Also the sizes of proteins considered meant that these were unable to determine the adsorption free energy, a crucial quantity in understanding the adsorption process, or to examine large-scale changes in protein conformation during adsorption.

In this paper molecular dynamics simulations are used to investigate the adsorption of model peptides onto nanostructured surfaces, specifically consisting of stripes containing hydrophilic and hydrophobic ligands. The peptides are examples of the so-called LK-peptides ${ }^{31}$, short sequences consisting of leucine (hydrophobic) and lysine (hydrophilic) residues. Depending on the spacing between the hydrophobic residues these form $\alpha$ helix or $\beta$-strands at hydrophobic interfaces. The surface was composed of a SAM consisting of alkylthiol molecules with differing functional groups on the outside. Alongside uniformly hydrophobic $\left(\mathrm{CH}_{3}\right.$-terminated $)$ and hydrophilic (OH-terminated) surfaces, nanostructured surfaces, consisting of stripes of hydrophobic and hydrophilic ligands, were constructed. The stripe widths vary between 1 and 5 molecules (approximately $4.3 \AA$ to 21.6 ̊). Using metadynamics simulations the adsorption strengths of the model peptides on these surfaces are determined, with the factors that control the adsorption strength investigated.

\section{SIMULATION DETAILS}

The simulated system consists of a single peptide molecule, in proximity to a self-assembled monolayer of varying functionality. Two different peptides are studied, these being $\operatorname{LK} \beta_{15}$ (LKLKLKLKLKLKLKL) and LK $\alpha_{14}$ (LKKLLKLLKKLLKL), which are designed sequences that form $\alpha$-helix $\left(\mathrm{LK} \alpha_{14}\right)$ or $\beta$-strands $\left(\mathrm{LK} \beta_{15}\right)$ at interfaces and surfaces ${ }^{31}$.

The surface consists of 280 alkylthiol $\left(\mathrm{SHC}_{11} \mathrm{H}_{22}-\mathrm{R}\right.$, with $\mathrm{R}=\mathrm{CH}_{3}$ or $\mathrm{OH}$ ) molecules, arranged in a $20 \times 14$ array. Surfaces are either homogeneous, solely containing either hydrophobic $\left(\mathrm{CH}_{3}\right)$ or hydrophilic $(\mathrm{OH})$ end groups, or striped, with widths of 5, 2, and 1 molecules. The different surfaces are denoted SAMch3, SAMoh, SAM5, SAM2, and SAM1, respectively. The chains were arranged in the $\sqrt{3} \times \sqrt{3}$ R3 geometry, using structures provided by the Latour research group (https://cecas .
clemson.edu/latourlabs/Jmol/Surfaces.html). OHterminated ligands were chosen for the hydrophilic component to avoid the strong electrostatic interactions that may arise for charged ligands. While the spacing between incompatible ligands may differ from this structure this simple model still allows us to investigate the effect of changing surface patterning. The positions of the terminal sulfur and hydrogen atoms are fixed to mimic the effect of the underlying surface. The peptide and SAM are solvated with approximately 9400 water molecules, with $\mathrm{Cl}^{-}$counter-ions added to neutralise the system. The system was periodic in the $x$ and $y$ directions while in the $z$-direction repulsive LJ walls were used.

To model the system the Charmm $36^{32-35}$ force field is used, with the charmm-variant of the TIP3P water mode $^{36}$ used for the water. Previous simulations have found that the Charmm family of force fields are capable of reproducing experimental values of the adsorption free energy $^{18}$.

The simulations were performed in the $N V T$-ensemble with the temperature controlled using a velocity rescaling algorithm ${ }^{37}$ (relaxation time $0.1 \mathrm{ps)}$ at $\mathrm{T}=300 \mathrm{~K}$. The simulation timestep was 2 fs. Bond lengths were constrained using the LINCS algorithm ${ }^{38}$. All systems were energy minimized using the steepest descent algorithm, followed by a short $N V T$ simulation (20 ps) with the heavy atoms in the proteins restrained to their initial positions using harmonic potentials with a force constant of $2.4 \mathrm{kcal} \mathrm{mol}^{-1} \AA^{-2}$. Following this a short $N V T$ simulation (20 ps), without restraining the heavy atoms, was performed.

For the calculation of the adsorption free energy welltempered metadynamics ${ }^{39}$ (MTD) combined with replica exchange with solute tempering ${ }^{40}$ (REST) was used. The use of metadynamics allows for the calculation of the free energy surface, with REST being used to enhance sampling of different peptide conformations. In the welltempered metadynamics simulations two collective variables were used for each set of simulations ${ }^{19}$. The first was the peptide centre-of-mass-surface separation. The surface was defined as the average $z$ position of the terminal heavy atom (either $\mathrm{C}$ or $\mathrm{O}$ ) in the alkylthiol chains.

The second collective variable was used to bias the peptide structure. For $\mathrm{LK} \alpha_{14}$ this $\mathrm{CV}$ is the number of $\alpha$ helical hydrogen bonds $\left(N_{\alpha-H B}\right)$, calculated from

$$
N_{\alpha-H B}=\sum_{i=1}^{N_{H B}} \frac{1-\left(r_{i} / r_{0}\right)^{n}}{1-\left(r_{i} / r_{0}\right)^{m}}
$$

where the sum runs over the $\alpha$-helical hydrogen bonds, $n=8, m=12$, and $r_{0}=2.5 \AA$. The switching function in Equation 1 goes from 1 as $r \rightarrow 0$ and 0 as $r \rightarrow \infty$ and is a continuous approximation to the Heaviside function.

For $\operatorname{LK} \beta_{15}$ the second CV is the dihedral offset given 
by

$D H=\frac{1}{2} \sum_{i=1}^{N-1}\left(1+\cos \left(\phi_{i}-\phi_{\text {ref }}\right)+\left(1+\cos \left(\psi_{i}-\psi_{\text {ref }}\right)\right)\right.$

where the sum runs over the residues in the peptide and $\phi_{i}$ and $\psi_{i}$ are the $\phi$ and $\psi$ angles of the $i$ th residue. The reference angles have values $\phi_{\text {ref }}=-2.36 \mathrm{rad}$ and $\psi_{\text {ref }}=2.36 \mathrm{rad}$, which correspond to an ideal $\beta$-strand with the leucine and lysine side chains being on opposite sides of the peptide backbone.

REST is a variation on replica exchange molecular dynamics ${ }^{41}$ where the temperature of only a subset of the system, in this case the protein, varies between replicas. This allows for the use of a smaller number of replicas compared to standard REMD. The temperature scaling is accomplished by scaling the peptide-peptide and peptidesystem interactions; the potential energy is given by

$$
E_{i}=\beta_{i} E_{p p}+\beta_{i}^{1 / 2} E_{p s}+E_{s s}
$$

where $E_{p p}$ is the peptide-peptide interaction, $E_{p s}$ is the interaction between the peptide and the remainder of the system (water and SAM), and $E_{s s}$ the interaction within the rest of the system. The scaling factor is given by $\beta_{i}=T_{0} / T_{i}$. In these simulations 12 replicas were used, with scaling factors $\beta_{i} 1.0(300 \mathrm{~K}), 0.955(314 \mathrm{~K}), 0.911$ $(329 \mathrm{~K}), 0.870(345 \mathrm{~K}), 0.830(361 \mathrm{~K}), 0.793(378 \mathrm{~K})$, $0.757(396 \mathrm{~K}), 0.722(415 \mathrm{~K}), 0.690$ (435 K), 0.658 (456 $\mathrm{K}), 0.629(477 \mathrm{~K})$, and $0.6(500 \mathrm{~K})$. The metadynamics weight function was allowed to evolve independently in each replica. A similar combination of REST with well tempered metadynamics was used to determine the adsorption free energy of small peptides onto $\mathrm{Ti}$ and $\mathrm{Si}^{42}$ and gold ${ }^{43}$ surfaces.

Exchanges between replicas were attempted every 500 timesteps (1 ps). The bias function was updated every 500 timesteps and a bias factor $\gamma=(T+\Delta T) / T=20$ was used. Following previous work the Gaussian height was $0.956 \mathrm{kcal} \mathrm{mol}^{-1}$ and the widths were $\sigma_{z}=0.1 \AA$, $\sigma_{a l p h a-H B}=0.4$, and $\sigma_{D H}=0.1$.

All simulations were performed using the Gromacs MD package (v4.6.7) $)^{44}$, using the PLUMED ${ }^{45,46}$ library for metadynamics and REST simulations. Standard gromacs tools were used to create the simulation input files. Analysis was performed using standard gromacs tools, $\mathrm{VMD}^{47}$, and with inhouse scripts using the MDAnalysis package $^{48}$.

Simulations were run for $200 \mathrm{~ns}$; convergence of the bias potential was determined by monitoring the RMSD in the bias potential taken at intervals of $10 \mathrm{~ns}$. For all simulations this was consistently below $0.01 \mathrm{kcal} \mathrm{mol}^{-1}$ at 200 ns. Following this 20 ns simulations with a constant bias potential were performed for each system, which were used to determine average properties (following removal of the bias potential). Specifically the average value of a property $X$ is calculated using

$$
\langle X\rangle=\frac{\sum_{i} X_{i} \exp \left[-\beta F\left(\left\{C V_{i}\right\}\right)\right]}{\sum_{i} \exp \left[-\beta F\left(\left\{C V_{i}\right\}\right)\right]}
$$

where $X_{i}$ is the value at $i$ th data set, $F$ is the free energy, $\left\{C V_{i}\right\}$ are the collective variables used to describe the peptide conformation, and $\beta=1 / k_{\mathrm{B}} T$. Uncertainties in these quantities were estimated using the standard deviation $\sigma_{X}=\sqrt{\left\langle X^{2}\right\rangle-\langle X\rangle^{2}}$.

\section{RESULTS}

\section{A. Peptide-surface Interaction}

Shown in Figure 1(a) are the free energy profiles for $\mathrm{LK} \beta_{15}$ and $\mathrm{LK} \alpha_{14}$. For both peptides strong adsorption is seen for the hydrophobic surface, consistent with previous simulation studies. While $\mathrm{LK} \beta_{15}$ shows only a single minimum near the surface, $\mathrm{LK} \alpha_{14}$ has three closely spaced minima of approximately equal depth arising due to different peptide conformations near the surface. Adsorption is significantly weaker for the $\mathrm{OH}$ terminated SAM; for $\mathrm{LK} \beta_{15}$ there is a shallow minimum $\left(\sim-15 \mathrm{kcal} \mathrm{mol}^{-1}\right)$ approximately $4 \AA$ from the surface. The interaction between $\mathrm{LK} \alpha_{14}$ and the SAMoh surface is largely repulsive. The values of adsorption free energy are similar to those determined in previous work ${ }^{18,19}$.

For the structured surfaces the free energy profile shows a strong dependence on the stripe width. The thickest stripes (SAM5) have free energy profiles similar to the uniformly hydrophobic surface. In this case it may be expected that the stripe is thick enough for the peptides to largely interact with the hydrophobic regions on the surface, minimising its exposure to the hydrophilic hydoxyl groups. As the stripes get thinner the depth of the free energy minimum decreases, getting closer to the uniformly hydrophilic surface.

The net adsorption free energy $\left(\Delta_{a d s} F\right)$ was calculated using $^{24}$

$$
\Delta_{a d s} F=-R T \ln \left(\frac{c_{a d s}}{c_{b u l k}}\right)
$$

where the adsorbed and bulk concentrations are given by

$$
\begin{aligned}
c_{a d s} & =\frac{1}{z_{0}-z_{\min }} \int_{z_{\min }}^{z_{0}} d z \exp [-\beta F(z)] \\
c_{\text {bulk }} & =\frac{1}{z_{\max }-z_{0}} \int_{z_{0}}^{z_{\max }} d z \exp [-\beta F(z)]
\end{aligned}
$$

where $z_{0}=10 \AA$. For both peptides $\Delta_{a d s} F$ is lowest for the hydrophobic methyl-terminated SAM, with the value of SAM5 similar (Figure 1(b)). As the stripe thickness decreases $\Delta_{a d s} F$ increases, indicating again that the adsorption strength of the peptides decrease as the surface structure goes to smaller sizes. This is consistent with experimental investigation of fibrinogen adsorption onto structured surfaces formed by bidentate 
(a)
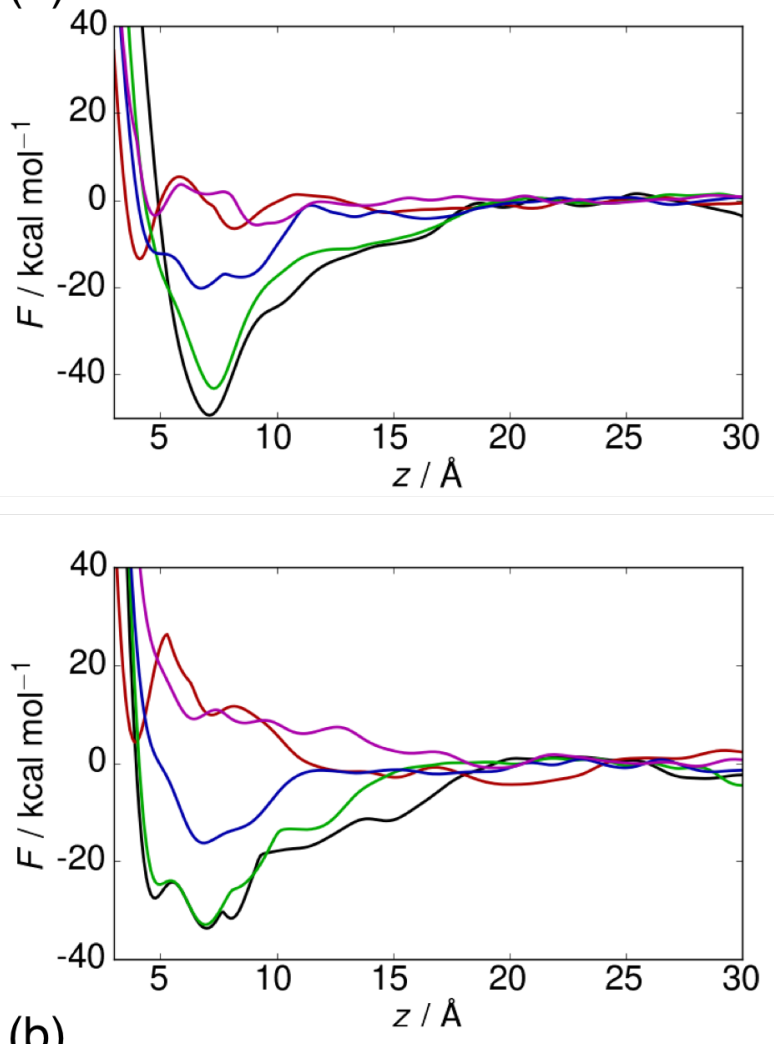

(b)

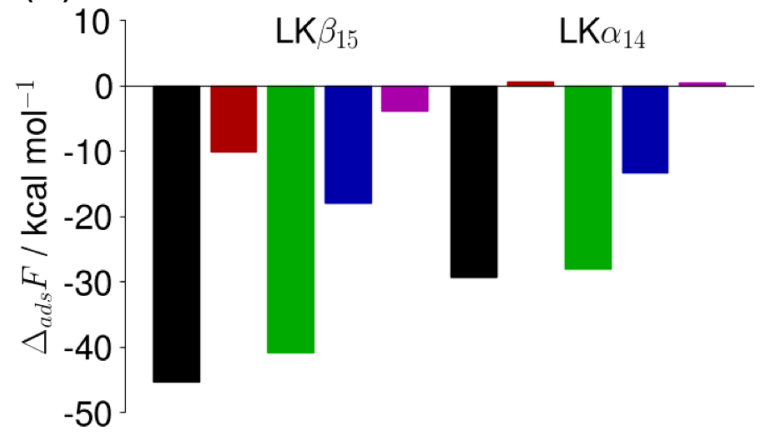

FIG. 1. (a) Free energy profiles for $L K \beta_{15}$ (top) and $L K \alpha_{14}$ (bottom) (b) net adsorption free energies (bottom). SAMch3, SAMoh, SAM5, SAM2, and SAM1 surfaces denoted by black, red, green, blue, and magenta respectively.

ligands with hydrophilic and hydrophobic end groups ${ }^{15}$. For the thinnest stripes $\Delta_{a d s} F$ is similar to that for the hydrophilic hydroxyl-terminated SAM.

Shown in Figure 2 are the free energy surfaces (FES) for the two peptides on the different surfaces. For LK $\beta_{15}$ on uniform surfaces there a minimum near the surface at large values of $D H$, corresponding to a conformation similar to a $\beta$-strand. This is expected for the $\mathrm{CH}_{3}$ terminated surface, where the LEU sidechains orient towards the hydrophobic surface and the LYS sidechains point towards the water. The minimum for the $\mathrm{OH}$-terminated surface is at lower peptide-surface separations, suggesting that the peptide is lying flatter against the surface. The free energy surface for the SAM5 surface is similar to that of the $\mathrm{CH}_{3} \mathrm{SAM}$, again showing that in this case the peptide largely interacts with the hydrophobic regions of the surface. For thinner stripes multiple minima appear, suggesting that a number of peptide conformations are found near the surfaces.

At the hydrophobic surface $\mathrm{LK} \alpha_{14}$ has a minimum at $N_{\alpha-H B} \sim 7.5$ showing that it adopts an $\alpha$-helical conformation near the surface. It also exhibits a second minimum at lower $N_{\alpha-H B}$ closer to the surface. This corresponds to a disordered conformation in contact with the surface. Similar behaviour is found for SAM5 and SAM2 surfaces with the minimum at large $N_{\alpha-H B}$ For both the OH-terminated and SAM1 surfaces the minimum free energy is in bulk solution for low $N_{\alpha-H B}$. In bulk solution we would expect a lower degree of $\alpha$-helix formation ${ }^{49}$ while near the hydrophilic surface the driving force for $\alpha$-helix formation compared to the hydrophobic surface.
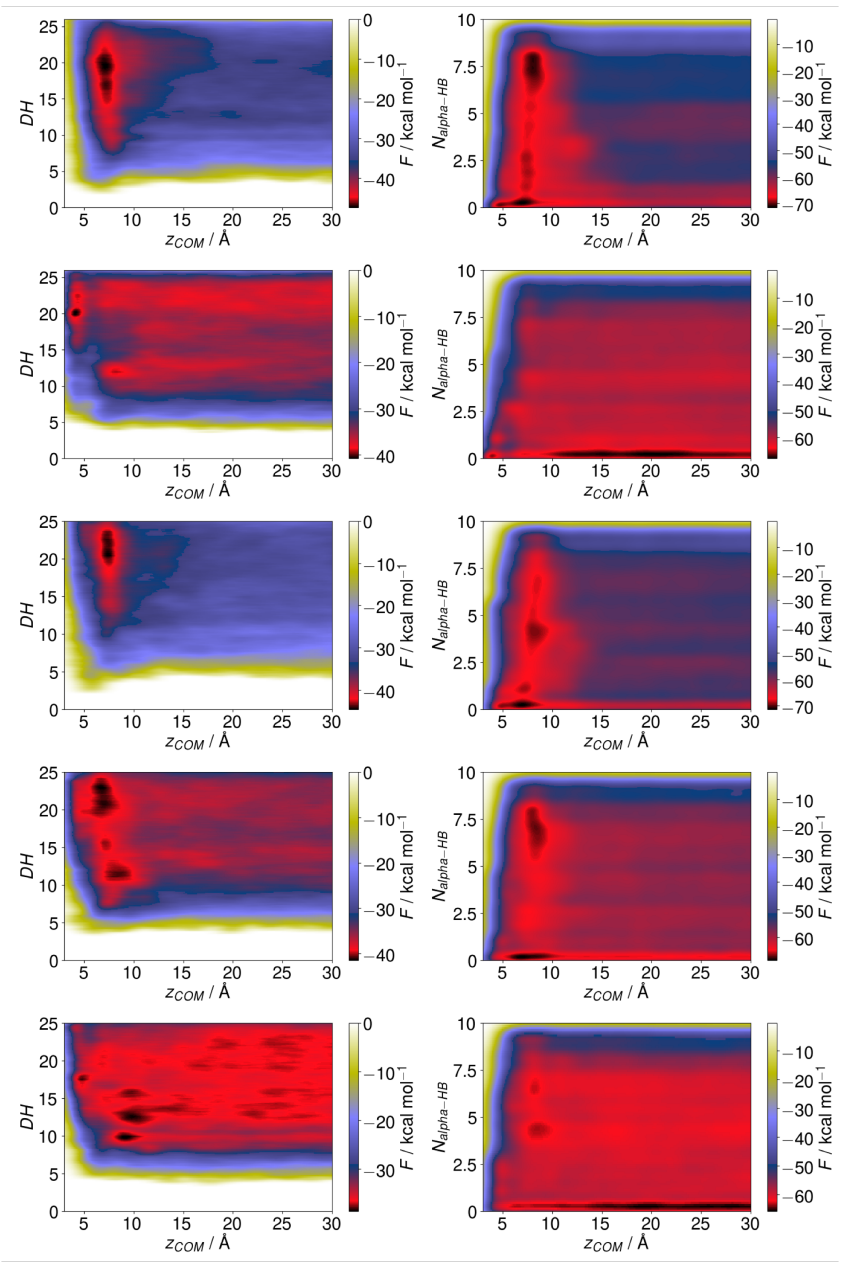

FIG. 2. Free energy surfaces for $L K \beta_{15}$ and $L K \alpha_{14}$ for (top to bottom) SAMch3, SAMoh, SAM5, SAM2, and SAM1 surfaces. 


\section{B. Peptide conformation}

The FES (Figure 2) suggest that both peptides exhibit multiple conformations at the surface. The conformation of the peptides at the surface can be investigated through the orientations of the amino acid sidechains (defined as the angle $\theta$ between the unit vector joining the $\mathrm{C} \alpha$ atom and the $\mathrm{C} \delta$ (LEU) or $\mathrm{N} \zeta$ (LYS) atoms and the $z$-axis). Shown in Figure 3 are the average $\cos \theta$ for each residue. The uniform surfaces (SAMch3 and SAMoh) behave consistently with previous experimental ${ }^{50}$ and computational $^{19}$ studies. For the SAMch3 surface the LEU sidechains typically point towards the surface (Figure 3) due to the hydrophobic interaction between these. For $\mathrm{LK} \beta_{15}$ this is reasonably uniform across the length of the peptide, while the LEU residue at the N-terminus of $\mathrm{LK} \alpha_{14}$, which preceeds two hydrophilic LYS residue, shows a reduced tendency to point towards the surface. The sidechain orientation for the SAMoh surface is less well-defined as the LYS sidechains which would be preferentially attracted to this surface are also well solvated in water. Rather, for both peptides, adsorption is mediated by leucine residues (L7 and L11 for $L K \beta_{15}$ and L5 and L12 for LK $\alpha_{14}$ ). For the thickest stripes (SAM5) the sidechain orientations are similar to the SAMch3 surface, with the LEU residues typically oriented towards the surface. Only for the central residues of $L K \beta_{15}$ (L7) are there significant differences. When the stripe width becomes smaller the side chain orientations for both peptides exhibit no strong regular ordering; in these cases the alternating hydrophilic and hydrophobic groups on the surface inhibit the formation of a well defined structure.

Changing surface structure may also change the overall shape of the peptides. Shown in Table 1 are the radii of gyration for the peptides on different surfaces. This was calculated only for cases where the peptide was in contact with the surface, using the criteria that the peptidesurface separation was less than $10 \AA$ (alternative definitions, such as ensuring at least one contact between a peptide and surface atom exists, give similar results). For $\mathrm{LK} \beta_{15} \quad R_{g}$ is lower for the uniform surfaces. The increase in the peptide size for the stripy surfaces may indicate a straightening out of the peptide to fit down the stripes. $\mathrm{LK} \alpha_{14}$ by contrast increases in size going form the SAMch3 to SAMoh surfaces, suggesting a distortion of the helical structure formed on the hydrophobic surface.

\begin{tabular}{ccc}
\hline & \multicolumn{2}{c}{$R_{g} / \AA$} \\
\hline Surface & $\mathrm{LK} \beta_{15}$ & $\mathrm{LK} \alpha_{14}$ \\
\hline SAMch3 & $9.7 \pm 1.0$ & $9.2 \pm 1.0$ \\
SAMoh & $9.7 \pm 0.6$ & $11.8 \pm 0.5$ \\
SAM5 & $10.7 \pm 0.1$ & $8.5 \pm 1.0$ \\
SAM2 & $10.5 \pm 1.0$ & $12.1 \pm 0.8$ \\
SAM1 & $10.0 \pm 1.5$ & $8.7 \pm 0.7$ \\
\hline
\end{tabular}

TABLE I. Radius of gyration for surface bound conformations.
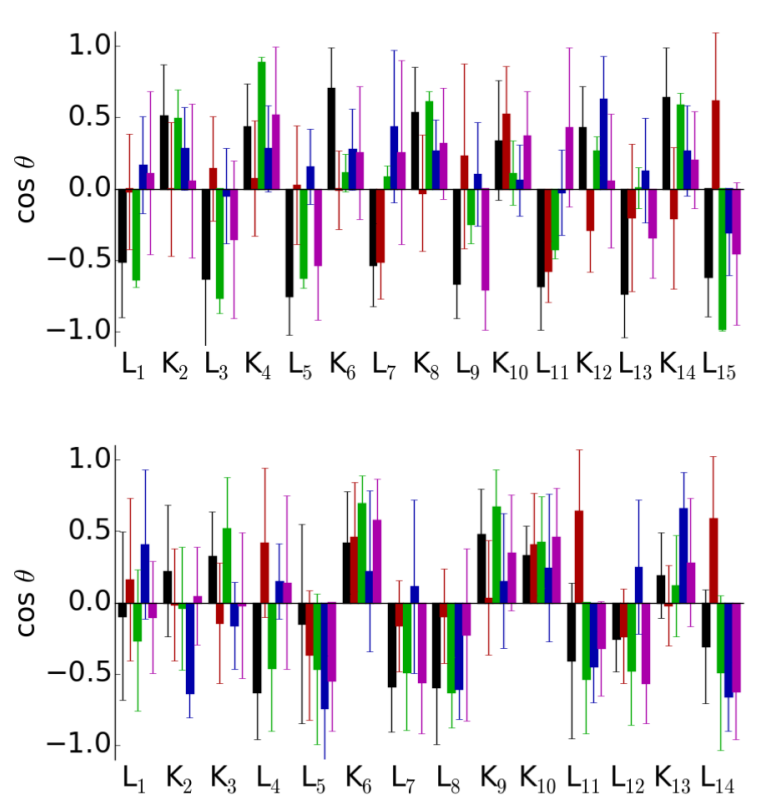

FIG. 3. Sidechain orientations for $L K \beta_{15}$ (top) and $L K \alpha_{14}$ (bottom) on SAM surfaces. Black, red, green, blue, and magenta denotes SAMch3, SAMoh, SAM5, SAM2, and SAM1 surfaces respectively.

More information on the effect of the surface structure on the peptide conformations can be found by determining the number of distinct peptide conformations. Using the method of Daura et al ${ }^{51}$ the surface bound peptide conformations were divided into clusters, based on the C $\alpha$ RMSD (with a cut-off of $3 \AA$ ). Shown in Table 2 are the number of distinct conformations found for each peptide and surface. The number of distinct conformations is typically larger for the uniform surfaces surfaces compared to the structured ones. This may arise due to the surface structure restricting the different conformations that the peptides can adopt. This decrease in the number of conformations leads to an additional entropic cost to adsorption compared to uniform surfaces ${ }^{52}$.

Visualisation of the most probable conformations (Figure 4) shows that the peptides preferentially adopt structures that maximise contact between hydrophobic residues and the methyl terminated chains. For SAMch3 this leads to the peptides adopting conformations that are flat on the surface, which for $\mathrm{LK} \alpha_{14}$ leads to distortions from a completely $\alpha$-helical structure. Similar behaviour is also seen for the SAM5 surface. For the other surfaces the peptides adopt conformations that typicailly have only a few residues are in contact with the surface. Only for $L K \beta_{15}$ on the SAM2 surface does the peptide lie flat on the surface.

The change in conformation entropy during adsorption can be estimated from the difference in conformational entropies for the surface and solvated conforma- 


\begin{tabular}{cccccc}
\hline Peptide & Surface & $N_{\text {cluster }}$ & $\Delta S / R$ & $S_{\text {surf }} / R$ & $S_{\text {solv }} / R$ \\
\hline \multirow{5}{*}{ LK $\beta_{15}$} & SAMch3 & 10 & 1.23 & 3.82 & 4.92 \\
& SAMoh & 12 & 0.04 & 4.02 & 3.98 \\
& SAM5 & 7 & 1.47 & 5.23 & 3.76 \\
& SAM2 & 4 & -2.05 & 2.94 & 4.99 \\
& SAM1 & 7 & -1.59 & 3.34 & 4.93 \\
\hline \multirow{5}{*}{$\mathrm{LK} \alpha_{14}$} & SAMch3 & 16 & -1.11 & 3.82 & 4.92 \\
& SAMoh & 9 & -1.84 & 3.06 & 4.90 \\
& SAM5 & 5 & -3.17 & 1.93 & 5.10 \\
& SAM2 & 8 & -0.49 & 3.94 & 4.42 \\
& SAM1 & 12 & -1.29 & 3.83 & 5.12 \\
\hline
\end{tabular}

TABLE II. Number of distinct surface bound conformations and entropy change between surface and solvated states.

tions. $S_{\text {surf }}$ and $S_{\text {solv }}$ can be found from the radius of gyration probability distributions ${ }^{53}$

$$
S_{i}=-k_{\mathrm{B}} \int d R_{g} p_{i}\left(R_{g}\right) \ln p_{i}\left(R_{g}\right) i=\operatorname{surf}, \text { solv }
$$

For $\operatorname{LK} \beta_{15} \Delta S$ is positive for the SAMch3 and SAM5 surfaces, where the peptide is largely in contact with hydrophobic regions of the surface (Figure 4), while the entropy change for the SAMoh surface is negligable (Table 2). By contrast $\Delta S$ is negative for these surfaces for $\mathrm{LK} \alpha_{14}$; its tendency to adopt $\alpha$-helical structures near hydrophobic surfaces restricts the probable conformations for the SAMch3 surface and the SAM5 surface, where the peptide largely contacts the hydrophobic regions of the surface. The conformational entropy decreases for the SAM2 and SAM1 surfaces again suggesting that the surface structure restricts the number of available conformations.

\section{Energetic contributions to peptide-surface interaction}

More insight into the factors that may determine adsorption strength can be found by considering the contributions to the peptide-surface interaction energies and the number of peptide-surface contacts (Table 3). For the uniform surfaces the interaction is stronger for the SAMoh surface than the SAMch3 surface, due to the stronger non-bonded interactions with the hydroxyl groups (especially as this has the fewest number of peptide-surface contacts). Energies for the SAMch3 and SAM5 surfaces are similar to each other, again demonstrating that the peptides are largely adsorbed onto the hydrophobic regions. For the SAM2 surface differences between the two peptides become apparent as the interaction for $L K \beta_{15}$ is significantly stronger than $L K \alpha_{14}$. This is consistent with the more extended conformation that LK $\beta_{15}$ adopts (Figure 4).

Considering the different types of peptide-surface contacts shows that leucine residues are more likely be in contact with the surface (contacts defined using the
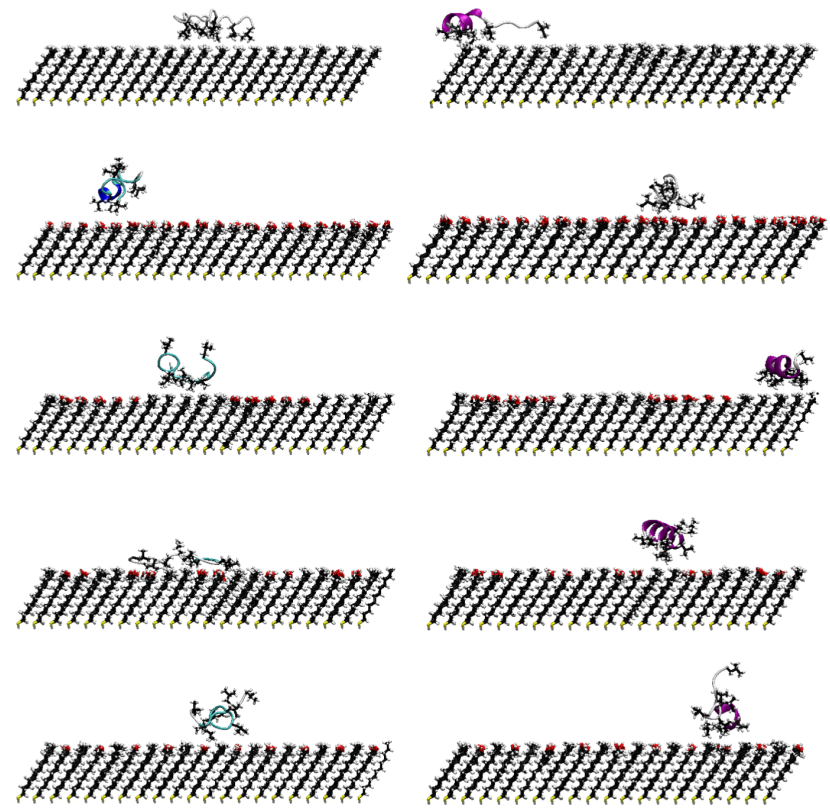

FIG. 4. Snapshots showing structures from the most probable clusters for $\mathrm{LK} \beta_{15}$ (left) and $\mathrm{LK} \alpha_{14}$ (right). From top to bottom SAMch3, SAMoh, SAM5, SAM2, and SAM1. Hydrophobic LEU sidechains are highlighted.

weight function in Equation 1 with $r_{0}=4.5 \AA$ ). Due to its amphiphilic side chain lysine contacts with both surface ligands ${ }^{30}$. The number of lysine- $\mathrm{CH} 3$ and lysine- $\mathrm{OH}$ contacts is approximately equal for the structured surfaces. For the thinnest stripes this is due to the close proximity between the different ligand types, while for the thicker (SAM5) stripes this may arise due to the peptide lying on the boundary between hydrophobic and hydrophilic surface regions.

\section{Water structure on mixed SAMS}

A large contribution to the anti-fouling behaviour of surfaces is thought to arise due to the presence of a dense water layer near the surface ${ }^{5,54}$. The density profile of water is shown in Figure 5(a). For the uniform surfaces there is a noticable peak in $\rho_{\text {water }}(z)$ near the SAM surface. The height of this peak is slightly higher for hydroxyl-terminated SAM, with this peak also occurring closer to the SAM surface. The structured surfaces show two peaks near the surface, corresponding to the water molecules close to the terminal hydroxyl and methyl groups. For all stripe widths the peak corresponding to the methyl-terminated molecules has approximately the same height and location. The peak corresponding to water molecules near hydroxyl groups gets larger but moves further away from the surface, gradually merging with 


\begin{tabular}{ccccccccc}
\hline Peptide & Surface & $E_{p s}$ & $E_{p s}^{v d w}$ & $E_{p s}^{\text {elec }}$ & $N_{\text {total }}$ & $N_{C H 3-L E U}$ & $N_{C H 3-L Y S}$ & $N_{O H-L Y S}$ \\
\hline \multirow{5}{*}{ LK $\beta_{15}$} & SAMch3 & $-18 \pm 8$ & $-20 \pm 8$ & $2.0 \pm 0.9$ & 56.1 & $51 \pm 8$ & $5 \pm 2$ & 0 \\
& SAMoh & $-29 \pm 15$ & $-15 \pm 6$ & $-14 \pm 11$ & 12 & 0 & 0 & $12 \pm 2$ \\
& SAM5 & $-15 \pm 1$ & $-16 \pm 2$ & $-0.8 \pm 0.2$ & 36 & $32 \pm 1$ & $2.9 \pm 0.5$ & $1.0 \pm 0.1$ \\
& SAM2 & $-94 \pm 37$ & $-50 \pm 17$ & $-44 \pm 20$ & 42 & $27 \pm 5$ & $7 \pm 2$ & $9 \pm 3$ \\
& SAM1 & $-19 \pm 11$ & $-16 \pm 6$ & $-4 \pm 6$ & 26 & $18 \pm 4$ & $4 \pm 1$ & $4 \pm 1$ \\
\hline \multirow{5}{*}{ LK $\alpha_{14}$} & SAMch3 & $-25 \pm 11$ & $-27 \pm 12$ & $2 \pm 1$ & 54 & $47 \pm 5$ & $6 \pm 3$ & 0 \\
& SAMoh & $-42 \pm 14$ & $-23 \pm 5$ & $-19 \pm 12$ & 13 & 0 & 0 & $13 \pm 3$ \\
& SAM5 & $-27 \pm 18$ & $-23 \pm 11$ & $-5 \pm 10$ & 47 & $41 \pm 4$ & $3 \pm 1$ & $3 \pm 2$ \\
& SAM2 & $-11 \pm 5$ & $-9 \pm 2$ & $-1 \pm 4$ & 23 & $17 \pm 2$ & $2 \pm 1$ & $3 \pm 1$ \\
& SAM1 & $-22 \pm 13$ & $-19 \pm 9$ & $-3 \pm 7$ & 27 & $21 \pm 6$ & $3 \pm 1$ & $3 \pm 1$ \\
\hline
\end{tabular}

TABLE III. Peptide-surface interaction energies (in $\mathrm{kcal} \mathrm{mol}^{-1}$ ) and numbers of peptide-surface contacts.

the other peak.
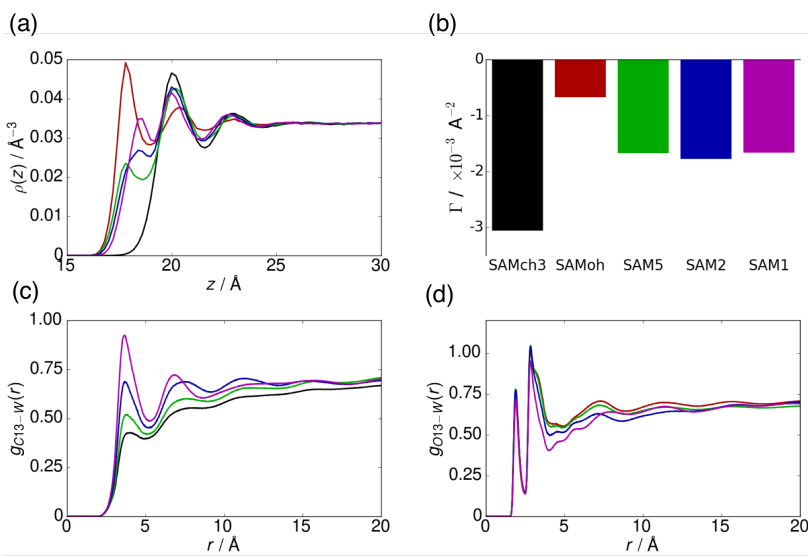

(d)

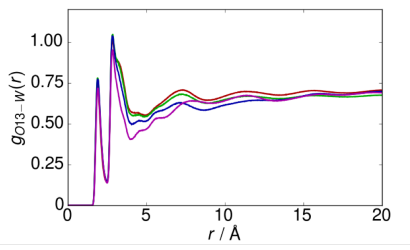

FIG. 5. Water structure near self-assembled monolayers. (a) Water density profile (b) Surface excess (c) Water-C13 radial distribution function (d) Water-O13 radial distribution function. In all cases SAMch3, SAMoh, SAM5, SAM2, and SAM1 are denoted by black, red, green, blue, and magenta respectively.

The amount of water adsorbed on the surface can be quantified through the adsorbance $(\Gamma)$

$$
\Gamma=\frac{1}{z_{2}-z_{1}} \int_{z_{1}}^{z_{2}} d z\left(\rho(z)-\rho_{b}\right)
$$

where $\rho(z)$ is the water density profile and $\rho_{b}$ is the bulk density of water. The integral runs from the peak in the density profile for the terminal heavy atoms in the $\operatorname{SAM}\left(z_{1} \approx 16.8 \AA\right)$ to $z_{2}=40 \AA$. As may be expected the adsorbance is highest for the hydrophilic hydroxylterminated SAM and lowest for the methyl-terminated SAM. For the mixed surfaces $\Gamma$ lies between these and is approximately the same for the different stripe widths. This suggests that the amount of water molecules near the surface plays only a minor role in determining the adsorption free energy for the structured surfaces.

The change in the water density profile and surface excess examine the water structure averaged over the

surface it is also useful to investigate how the structure around the hydrophobic and hydrophilic parts of the surface changes with the stripe thickness. As the stripe width decreases the peak on the C13-water RDF (Figure 5(c)) increases due to water molecules that are close to hydroxyl groups in the $\mathrm{OH}$-terminated molecules. The O13-water RDF shows less variation with the stripe width (Figure 5(d)), due to the strong interaction between water molecules and the $\mathrm{OH}$ groups.

Differences in the water structure are also reflected in changes to the number of hydrogen bonds between water molecules and the surface (Table 4). The average number of water-surface hydrogen bonds is for SAMoh surface is approximately twice that of SAM5 and SAM2, reflecting the larger number of $\mathrm{OH}$ groups in the uniform SAMoh surface. For the SAM1 surface the number of hydrogen bonds is significantly smaller than for the other mixed surfaces as the narrower stripes hinder the formation of hydrogen bonds. Notably the number of hydrogen bonds is essentially unchanged when the peptide is at the surface.

\begin{tabular}{cccc}
\hline Surface & $N_{\text {hbond }}$ & $N_{\text {hbond }}\left(\mathrm{LK} \beta_{15}\right)$ & $N_{\text {hbond }}\left(\mathrm{LK} \alpha_{14}\right)$ \\
\hline SAMoh & $620 \pm 14$ & $625 \pm 15$ & $621 \pm 14$ \\
SAM5 & $325 \pm 10$ & $317 \pm 7$ & $325 \pm 10$ \\
SAM2 & $321 \pm 10$ & $304 \pm 12$ & $315 \pm 9$ \\
SAM1 & $281 \pm 9$ & $279 \pm 9$ & $280 \pm 9$ \\
\hline
\end{tabular}

TABLE IV. Number of hydrogen bonds between surface and water.

\section{CONCLUSIONS}

Using molecular dynamics simulations, employing advanced sampling methods, we investigated the interaction between peptides and nanostructured surfaces. The adsorption strengths were found to depend both on the chemistry of the surface (changing from hydrophobic to hydrophilic) and the surface structure. Strongest adsorption is found for surfaces with larger hydrophobic regions as this allows for the hydrophobic interactions with the 
LEU sidechains. As the stripe widths get smaller the adsorption strength decreases, with it becoming similar to that for a uniformly hydrophilic surface for the thinnest stripes. The free energy surfaces (Figure 2) shows that the uniform hydrophobic surface has only a few free energy minima near the surface. This also holds for the mixed surface with broad stripes, where the peptides can largely avoid contact with hydrophilic ligands. As the stripes get narrower the FES exhibits multiple, shallow minima as the peptides try to adopt conformations commensurate with the surface structure. Qualitative, as well as quantitative, differences were found between the two peptides studied, showing that the interplay between peptide and surface structures affects the adsorption behaviour.

Adsorption of peptides onto the surface leads to changes in their conformation. For surfaces with larger hydrophobic regions (SAMch3 and SAM5) the peptide conformation can be rationalized through the hydrophobic leucine side chains pointing towards the hydrophobic surface. This conformation becomes disrupted as the stripes get narrower. Consideration of the number of peptide conformations at the surface suggests that the peptide conformational entropy plays a role in determining how the adsorption strength depends on the surface structure. By contrast only small changes are seen in the water structure for the different mixed SAMs so this is less likely to play a role (although it may be important in determining the differing adsorption strengths between the hydrophibic and hydrophilic surfaces).

Both components of the systems studied in this work, the peptides and surfaces, represent idealised model systems. Nonetheless it has revealed details of the relationship between nanoscale surface structure and protein adsorption strength. In future work this will be extended to consider more realistic models of the surface, along with different realisations of surface heterogeneity. Likewise it would be useful to consider more complex proteins, to give more information on the interplay between protein and surface structure. This would give insight into the use of nanostructured surfaces in prevention of protein adsorption in biomaterials and antifouling applications, as well as linking surface structure to the functionality of adsorbed proteins and formation of supramolecular protein structures at surfaces.

\section{ACKNOWLEDGEMENTS}

I wish to acknowledge the SFI/HEA Irish Centre for High-End Computing (ICHEC) for the provision of computational facilities and support.
* david.cheung@nuigalway.ie

1 I. Banerjee, R. C. Pangule, and R. S. Kane, Advanced materials 23, 690 (2011).

2 C. Blaszykowski, S. Sheikh, and M. Thompson, Chemical Society Reviews 41, 5599 (2012).

3 M. Mrksich and G. M. Whitesides, Annual review of biophysics and biomolecular structure 25, 55 (2003).

${ }^{4}$ N. Ayres, Polym. Chem. 1, 769 (2010).

5 S. Chen, L. Li, C. Zhao, and J. Zheng, Polymer 51, 5283 (2010).

${ }^{6}$ C. Rodriguez-Emmenegger, E. Brynda, T. Riedel, M. Houska, V. Šubr, A. B. Alles, E. Hasan, J. E. Gautrot, and W. T. S. Huck, Macromolecular Rapid Communications 32, 952 (2011).

7 R. E. Holmlin, X. Chen, R. G. Chapman, S. Takayama, and G. M. Whitesides, Langmuir 17, 2841 (2001).

8 S. Chen, F. Yu, Q. Yu, Y. He, and S. Jiang, Langmuir 22, 8186 (2006).

9 G. Pace, A. Petitjean, M. N. Lalloz-Vogel, J. Harrowfield, J. M. Lehn, and P. Samorì, Angewandte Chemie - International Edition 47, 2484 (2008).

10 A. R. Puente Santiago, T. Pineda, M. Blázquez, and R. Madueño, Journal of Physical Chemistry C 120, 8595 (2016).

11 K.-i. Iimura, T. Shiraku, and T. Kato, Langmuir 18, 10183 (2002).

12 A. R. Puente Santiago, G. Sánchez-Obrero, T. Pineda, M. Blázquez, and R. Madueño, Journal of Physical Chemistry C 122, 2854 (2018).

13 E. Xi, V. Venkateshwaran, L. Li, N. Rego, A. J. Patel, and S. Garde, Proceedings of the National Academy of Sciences p. $201700092(2017)$.

14 H. J. Lee, A. C. Jamison, and T. R. Lee, Chemistry of Materials 28, 5356 (2016).

15 P. Chinwangso, H. J. Lee, A. C. Jamison, M. D. Marquez, C. S. Park, and T. R. Lee, Langmuir 33, 1751 (2017).

16 M. D. Marquez, O. Zenasni, A. C. Jamison, and T. R. Lee, Langmuir 33, 8839 (2017).

17 J. J. Kuna, K. Voïtchovsky, C. Singh, H. Jiang, S. Mwenifumbo, P. K. Ghorai, M. M. Stevens, S. C. Glotzer, and F. Stellacci, Nature materials 8, 837 (2009).

18 G. Collier, N. A. Vellore, J. A. Yancey, S. J. Stuart, and R. A. Latour, Biointerphases 7, 24 (2012).

19 M. Deighan and J. Pfaendtner, Langmuir : the ACS journal of surfaces and colloids 29, 7999 (2013).

20 D. Mallinson, D. L. Cheung, D. Simionesie, A. B. Mullen, Z. J. Zhang, and D. A. Lamprou, Journal of Biomedical Materials Research - Part A 105, 737 (2017).

21 M. Ozboyaci, D. B. Kokh, S. Corni, and R. C. Wade, Quarterly Reviews of Biophysics 49, 1 (2016).

22 T. R. Walsh, Accounts of Chemical Research 50, 1617 (2017).

23 J. Zheng, L. Li, S. Chen, and S. Jiang, Langmuir 20, 8931 (2004).

24 C. P. O' Brien, S. J. Stuart, D. A. Bruce, and R. A. Latour, 24, 14115 (2008).

25 P. K. Biswas, N. A. Vellore, J. A. Yancey, T. G. Kucukkal, G. Collier, B. R. Brooks, S. J. Stuart, and R. A. Latour, Journal of computational chemistry 33, 1458 (2012).

26 Y. Xie, M. Liu, and J. Zhou, Applied Surface Science 258, 8153 (2012). 
27 Z. A. Levine, S. A. Fischer, J. E. Shea, and J. Pfaendtner, Journal of Physical Chemistry B 119, 10417 (2015).

28 J. Liu and J. Zhou, Acta Biomaterialia 40, 23 (2016).

29 A. Hung, S. Mwenifumbo, M. Mager, J. J. Kuna, F. Stellacci, I. Yarovsky, and M. M. Stevens, Journal of the American Chemical Society 133, 1438 (2011).

30 M. Penna, K. Ley, S. Maclaughlin, and I. Yarovsky, Faraday Discuss. 191, 435 (2016).

31 W. DeGrado and J. Lear, Journal of the American Chemical Society 107, 7684 (1985).

32 A. D. MacKerell, D. Bashford, R. L. Dunbrack, J. D. Evanseck, M. J. Field, S. Fischer, J. Gao, H. Guo, S. Ha, D. Joseph-McCarthy, et al., The Journal of Physical Chemistry B 102, 3586 (1998).

33 A. D. Mackerell, M. Feig, and C. L. Brooks, Journal of Computational Chemistry 25, 1400 (2004).

34 P. Bjelkmar, P. Larsson, M. A. Cuendet, B. Hess, and E. Lindahl, J. Chem. Theory Comput. 6, 459 (2010).

35 R. B. Best, X. Zhu, J. Shim, P. E. M. Lopes, J. Mittal, M. Feig, and A. D. MacKerell Jr, Journal of Chemical Theory and Computation 8, 3257 (2012).

36 P. Mark and L. Nilsson, Journal of Physical Chemistry A 105, 9954 (2001).

37 G. Bussi, D. Donadio, and M. Parrinello, Journal of Chemical Physics 126, 014101/1 (2007).

38 B. Hess, H. Bekker, H. J. C. Berendsen, and J. G. E. M. Fraaije, Journal of computational chemistry 18, 1463 (1997).

39 A. Barducci, G. Bussi, and M. Parrinello, Physical Review Letters 100, 020603 (2008).

40 P. Liu, B. Kim, R. A. Friesner, and B. J. Berne, Proceedings of the National Academy of Sciences of the United
States of America 102, 13749 (2005).

41 Y. Sugita and Y. Okamoto, Chemical Physics Letters 314, 141 (1999).

42 J. Schneider and L. Colombi Ciacchi, Journal of the American Chemical Society 134, 2407 (2012).

${ }^{43}$ L. B. Wright, J. P. Palafox-Hernandez, P. M. Rodger, S. Corni, and T. R. Walsh, Chem. Sci. 6, 5204 (2015).

44 B. Hess, C. Kutzner, D. van der Spoel, and E. Lindahl, J. Chem. Theory Comput. 4, 435 (2008).

45 G. A. Tribello, M. Bonomi, D. Branduardi, C. Camilloni, and G. Bussi, Computer Physics Communications 185, 604 (2014).

46 G. Bussi, Molecular Physics 112, 379 (2013).

47 W. Humphrey, A. Dalke, and K. Schulten, Journal of Molecular Graphics 14, 33 (1996).

${ }^{48}$ N. Michaud-Agrawal, E. J. Denning, T. B. Woolf, and O. Beckstein, Journal of computational chemistry 32, 2319 (2011).

49 C. Dalgicdir, C. Globisch, C. Peter, and M. Sayar, PLOS Computational Biology 11, e1004328 (2015).

50 T. Weidner and D. G. Castner, Physical chemistry chemical physics : PCCP 15, 12516 (2013).

51 X. Daura, K. Gademann, B. Jaun, D. Seebach, W. F. Van Gunsteren, and A. E. Mark, Angew. Chemie Int. Ed. 38, 236 (1999).

52 S. Corni, Jom 67, 781 (2015).

53 S. R. Euston, Biomacromolecules 11, 2781 (2010).

54 S. Sheikh, C. Blaszykowski, R. Nolan, D. Thompson, and M. Thompson, Journal of Colloid and Interface Science 437, 197 (2015). 